\title{
Medición del conocimiento adquirido en tecnología de la madera por industriales forestales en Costa Rica
}

\section{Measuring knowledge acquired in wood technologies by forest industries in Costa Rica}

Luis Diego Camacho-Cornejo', Cynthia Salas-Garita², Lupita VargasFonseca $^{3}$, Juan Carlos Valverde-Otárola ${ }^{4}$

Fecha de recepción: 21 de octubre de 2016 Fecha de aprobación: 25 de enero de 2017

Camacho-Cornejo, L; Salas-Garita, C; Vargas-Fonseca, L; Valverde-Otárola, J. Medición del conocimiento adquirido en tecnología de la madera por industriales forestales en Costa Rica. Tecnología en Marcha. Vol. 30-3. Julio-Setiembre 2017.

Pág 35-46.

DOI: 10.18845/tm.v30i3.3271

1 Profesor-Investigador. Escuela de Ingeniería Forestal, Instituto Tecnológico de Costa Rica. Costa Rica. Correo Electrónico: dicamacho@itcr.ac.cr.

2 Profesora-Investigadora. Escuela de Ingeniería Forestal, Instituto Tecnológico de Costa Rica. Costa Rica. Correo Electrónico: cysalas@itcr.ac.cr.

3 Profesora-Investigadora. Escuela de Ingeniería Forestal, Instituto Tecnológico de Costa Rica. Costa Rica. Correo Electrónico: Ivargas@itcr.ac.cr.

4 Investigador. Escuela de Ingeniería Forestal, Instituto Tecnológico de Costa Rica. Costa Rica. Correo Electrónico: jcvalverde@itcr.ac.cr. 


\title{
Palabras clave
}

Aprendizaje; cuantificación de conocimiento; secado madera; preservado madera; normas de calidad.

\section{Resumen}

La cuantificación del conocimiento adquirido en individuos de edad adulta, permite no solo definir la efectividad de un sistema de aprendizaje, sino que también abre la posibilidad de definir las fortalezas y debilidades del sistema y analizar mejoras para el aumento de la retención de conocimiento; por lo cual el objetivo de este estudio consistió en cuantificar el conocimiento adquirido en tres temáticas de tecnología de la madera dirigidas a industriales forestales de Costa Rica. Se trabajó con cinco grupos focales de distintas zonas del país, a los cuales se les proporcionó conocimiento en tres temáticas: secado, preservado y normas de calidad en madera, impartidas mediante un sistema de aprendizaje significativo-observacional, evaluando el conocimiento en tres etapas: previo e inmediatamente posterior a la impartición del curso y 30 semanas postcurso. Los resultados mostraron que el sistema empleado fue funcional en el tema de secado, el cual expuso retenciones de conocimiento superiores al $70 \%$ con la percepción por parte del grupo de estudio de que el tema se impartió de forma muy funcional y simple; respecto al tema preservado se presentó una retención del 62,9 \% y una funcionalidad aceptable del sistema, con la observación de que se debe mejorar la técnica de impartición del conocimiento teórico, factor que el grupo denotó como complicado y que incidió en una retención teórica baja. Finalmente, el tema de la norma de calidad, no mostró una funcionalidad óptima bajo el sistema de aprendizaje aplicado, presentando la menor retención del conocimiento (54,7 \%) y una opinión muy dividida del grupo en estudio acerca de su funcionalidad y simplicidad, sugiriendo la necesidad de valora un cambio de sistemas de aprendizaje o re evaluación del sistema empleado.

\section{Keywords}

Learning quantification of knowledge; drying wood; preserved wood; quality standards.

\begin{abstract}
Quantification of knowledge acquired in adulthood individuals, can not only define the effectiveness of a learning system, but also opens the possibility of defining the strengths and weaknesses of the system and analyze improvements to increase knowledge retention; so the aim of this study was to quantify the knowledge acquired in three thematic wood technology aimed at forest industry of Costa Rica. We worked with five focus groups in different areas of the country, which were provided knowledge on three themes: dried, preserved and quality standards wood, delivered through a system of significant-observational learning, assessing knowledge in three stages: prior and immediately following the delivery of the course and 30 weeks post-course. The results showed that the system used was functional in the subject drying, which exhibited superior knowledge withholding $70 \%$ with the perception by the study group was given the issue very functional and simple; preserved issue regarding retention of $62.9 \%$ and an acceptable system functionality, with the observation that should improve the teaching of theoretical knowledge which the group denoted as complicated and influenced a low theoretical retention was presented. Finally, the issue of quality standard, showed optimal functionality with the system applied learning, presenting the slightest knowledge retention $(54.7 \%)$ and a very divided opinion of the study group functionality and simplicity suggesting the need for values a change or re-learning systems evaluation system used.
\end{abstract}




\section{Introducción}

El aprendizaje es el proceso en el cual un individuo adquiere conocimientos [1]-[3], habilidades [4], [5] y actitudes [1], [4] a partir de enseñanza directa (generada por experiencias personales) o indirecta (transmitida por otro individuo) en el tiempo; la medición del aprendizaje consiste en el desarrollo de herramientas que permitan definir cuantitativa y cualitativamente la cantidad de información que el usuario efectivamente adaptó en su razonamiento [4], [5]. Uno de los aspectos de mayor inferencia en el aprendizaje y su evaluación es la edad [5], [17] al inferir en cambios en la capacidad de asimilar conceptos por parte de un individuo; factores como la receptibilidad neurológica, capacidad de asimilación, conciencia de entendimiento y capacidad de retención [6], [18]. Siendo necesario variar significativamente las técnicas y los sistemas de medición de conocimiento adquirido [19].

En el caso específico de la retención de conocimiento de la etapa adulta comprendida de los 20 a 60 años, estudios desarrollados por Kashiwaba et al. [20] con varios grupos étnicos a nivel mundial, han encontrado que uno de los sistemas de aprendizaje que mejor respuesta ha presentado es el significativo-observacional, por la combinación de conceptos teóricos y actividades prácticas que aceleran la capacidad del entendimiento de información y con ello el tiempo de aprendizaje se disminuye y la retención de información en el mediano plazo es elevada; sin embargo, se presentan limitaciones en su valoración cuantitativa, debido a que aspectos como la variabilidad de conocimientos previos de los individuos en conjunto a perjuicios previos y factores exenticos como el estrés, cargas emocionales y limitaciones de tiempo hacen que la evaluación no sea óptima y en muchos casos engañosa, por tanto se deben considerar en la evaluación para que esta pueda minimizar estos efectos en las cuantificación [5]-[8].

En los sistemas de evaluación del conocimiento adquirido como en el sistema significativoobservacional se destaca la evaluación memorística, que se basa en la aplicación periódica de pruebas y consulta de temas o aspectos específicos o procedimientos prácticos, siendo un sistema de control continuo, pero no garantiza la retención de información en el mediano plazo [7]; otro sistema es la evaluación mixta que combina simultáneamente aspectos teóricos y prácticos en la evaluación, permitiendo generar perfiles de aprendizaje amplios y especificar qué áreas o aspectos del individuo han tenido un aprendizaje bajo o ineficiente, la limitación de este sistema es la complejidad de su ejecución [8], recientemente se ha incluido la evaluación humanista que considera una vinculación de evaluaciones de elementos emocionales, que permite ver el grado de relación de la capacidad de síntesis de información con el estado emocional que le genera, con ello desarrollar aprendizajes de forma más placentera, la limitación actual de este sistema, ha sido su complejidad de desarrollo [7], [18].

En el caso específico del aprendizaje y su cuantificación en temáticas forestales, la FAO [21] menciona que es un tema prioritario en esta década, al ser el impacto social que tiene la trasmisión de conocimientos de manejo del recurso forestal un potencial de desarrollo en las comunidades; sin embargo, actualmente, mundial el aprendizaje no ha sido el idóneo, ya que muchas de las técnicas tradicionales de enseñanza implementadas carecen de herramientas reales de cuantificación del aprendizaje, involucrando con ello problemas al definir los elementos de enseñanza que deben ser mejorados, la razón de esto se ha debido a que muchos de los profesionales en ciencias forestales no cuentan con las herramientas adecuadas de aprendizaje o las metodologías implementadas se limitan a la transmisión del conocimiento de una manera más teórica y desconectada con la realidad de la región. Por tanto, se planteó como objetivo del presente estudio cuantificar el conocimiento adquirido en tres temáticas de tecnología de la madera dirigidas a industriales forestales de Costa Rica. 


\section{Materiales y métodos}

\section{Temáticas desarrolladas y grupo de estudio}

El proceso de aprendizaje se desarrolló con tres temas: el secado (definido como el proceso en el cual se extrae el agua de la madera [9]), preservado (proceso en que se aplican químicos para aumentar la durabilidad del material en el tiempo [10]) y norma de calidad de la madera INTE 06-07-01:2011 (Terminología de maderas [22] y INTE 06-07-02:2014 para Madera aserrada para uso general [23]) en diez especies forestales (Acacia mangium, Alnus acuminata, Bombapcosis quinata, Cupressus Iusitanica, Gmelina arborea, Swietenia macrophylla, Tectona grandis, Terminalia amazonia, Terminalia oblonga y Vochysia guatemalensis) utilizadas actualmente en proyectos de reforestación comercial, consideradas en el manejo de bosques naturales y en el desarrollo de productos maderables [24]. En las temáticas se abarcaron aspectos como los tipos de secado y preservado existentes, aplicabilidad de la normativa, sus características, ventajas y desventajas, además, la existencia de normas que los evalúen y como se implementan estas en sistemas productivos.

El proceso de aprendizaje se realizó en cinco regiones del país (Zona Norte, Atlántica, Sur, Valle Central y Guanacaste) y se dirigió a grupos con un promedio de quince participantes, que se caracterizaban por tener edades de 25 a 50 años, de ambos géneros y con formación en ciencias forestales o bien con experiencia en el desarrollo de actividades forestales con fines industriales y comerciales. El proceso de selección de dichos participantes se dio en función a su disposición en participar en todas las actividades del curso y estar ligados en actividades laborales que vincularan los conocimientos de ambos temas.

\section{Proceso de aprendizaje implementado}

El proceso de aprendizaje implementado fue del tipo significativo-observacional [20] que consistió en dos fases: la primera la impartición de clases magistrales sobre terminologías y conceptos básicos en los temas de secado y preservado de madera y la segunda fase consistió en el desarrollo de actividades grupales prácticas en las que se aplicaba el proceso de evaluación y operación del secado y preservado de la madera, así como una guía supervisada del control y evaluación de la calidad de la madera.

\section{Evaluación de aprendizaje}

Se desarrolló tres fases de evaluación del proceso de aprendizaje, la primera se aplicó previo a la impartición del conocimiento de cada una de las temáticas, la segunda se realizó una vez implementada la lección teórica y práctica de cada tema y finalmente se desarrolló 30 semanas posterior a la finalización el curso; estas evaluaciones se realizaron al mismo grupo de estudio y se utilizó los aspectos mostrados en el cuadro 1, considerando: conocimiento base del usuario, conocimiento teórico y conocimiento práctico.

Análisis estadístico

La caracterización previa del curso se desarrolló a partir de un análisis descriptivo de conocimiento previo, habilidades prácticas de la temática, interés de aprendizaje y percepción de dificultad. Para definir si existía significancia en los resultados se realizó un análisis de varianza de una vía, en el caso de presentar diferencias significativas en los resultados se aplicó la prueba de Tukey. La Curva de conocimiento adquirido cuantifico (CAC) se desarrolló con los datos adquiridos del precurso, postcurso y 30 semanas post curso, se generó a partir de la ecuación 1, en la cual conforme el valor del CAC tienda a 100 la información cuantificable asimilada es mayor. 
Donde:

$$
C A C(\%)=\left(\left(V A C_{\text {teórico }}+V A C_{\text {práctico }}\right) / 2\right) \cdot 100 \%
$$

CAC: $\quad$ es el valor porcentual de la Curva de conocimiento adquirido cuantifico

$V_{A C} C_{\text {teórico: }}$ Valores de aprendizaje cuantificado teórico

$V_{A C}$ práctico: Valores de aprendizaje cuantificado práctico

Los VAC teóricos y prácticos se generaron a partir de la ecuación 2, que se obtuvo de los estudios previos desarrollados [11]-[13] ,

$$
V A C_{t / p}=C P \cdot 0,1+A A_{t / p} \cdot 0,25+C R C_{t / p} \cdot 0,15
$$

Donde:

$V_{\mathrm{VAC}} \quad$ es el valor de aprendizaje cuantificado teórico o práctico.

$\mathrm{CP} \quad$ es el conocimiento previo

$\mathrm{AA}_{\mathrm{t} / \mathrm{p}} \quad$ es el aprendizaje adquirido teórico o práctico

$\mathrm{CRC}_{\mathrm{t} / \mathrm{o}} \quad$ es la capacidad de razonamiento crítico (capacidad entendimiento real de lo aprendido) teórico o práctico

El análisis perceptual de los cursos (tanto general como la sección teórica y práctica), se realizó bajo los términos de utilidad (utilidad elevada-utilidad baja) y complejidad (simple-complicado), para lo cual se utilizó un análisis tipo SANN (Analysis/Deployment) a partir del principio de clasificación de VAC teórico y práctico. Los análisis estadísticos se realizaron con los programas STATISTICA versión 8,9 y R versión 3.3.1 con un nivel de significancia de 0,05.

\begin{tabular}{|c|c|}
\hline Sección funcional & Aspecto evaluado \\
\hline \multirow{3}{*}{$\begin{array}{c}\text { Conocimiento base del } \\
\text { usuario }\end{array}$} & Conoce la definición de secado/preservado \\
\hline & Conoce la variación de secado /preservado por especies madera \\
\hline & Conoce utilidad de la actividad de secado/preservado \\
\hline \multirow{3}{*}{$\begin{array}{l}\text { Conocimiento teórico } \\
\text { del usuario }\end{array}$} & Dominio de tecnicismos de las operaciones de secado/preservado \\
\hline & Entendimiento de valores o parámetros generados en las actividades \\
\hline & Diferenciación y entendimiento de distintos sistemas de secado/preservado \\
\hline \multirow{4}{*}{$\begin{array}{c}\text { Conocimiento práctico } \\
\text { del usuario }\end{array}$} & Como desarrolla actividades de secado/preservado \\
\hline & Proceso de control del secado/preservado \\
\hline & Reconocimiento de la calidad de la actividad realizada \\
\hline & Aplicación práctica de control de normas de calidad \\
\hline
\end{tabular}

Cuadro 1. Variables evaluadas en el proceso de aprendizaje aplicado en tres temáticas industriales forestales. 


\section{Resultados}

\section{Caracterización cuantitativa de grupo de estudio previo a los cursos}

La evaluación previa del conocimiento al grupo estudiado (cuadro 2) mostró que el conocimiento de la temática de secado y preservado es similar, en cambio la norma de calidad de madera presentó valores significativamente distintos. Los resultados mostraron un nivel bajo de conocimientos básicos (inferiores al 50\%), pero con una aplicabilidad de las temáticas en las actividades laborales diarias elevada para el caso de secado y preservado (mayor al 60\%) y baja con la norma calidad (45,5\%), evidenciando una aplicabilidad laboral significativa de las temática pero con ausencia de conocimientos verídicos que la sustenten; en contra parte al evaluar el interés de adquisición del conocimiento, el grupo presentó un grado de interés superior al 90\% considerado como muy alto pero con la premisa de que la precepción de la temática de la norma de calidad podría ser significativamente más difícil (60,8 \%) que los temas de secado y preservado, los cuales no mostraron diferencias entre sí (entre 30 y 45\%).

Cuadro 2. Valores medio de los parámetros considerados en la línea base de conocimientos del grupo de estudio aplicado con tres temáticas industriales forestales.

\begin{tabular}{|c|c|c|}
\hline Parámetro considerado & Temática & Valor porcentual \\
\hline \multirow{3}{*}{ Conocimiento básico de la temática } & Secado & $56,5^{\mathrm{a}}(10,5)$ \\
\hline & Preservado & $56,4^{\mathrm{a}}(11,6)$ \\
\hline & Norma de calidad & $33,3^{b}(15,7)$ \\
\hline \multirow{3}{*}{$\begin{array}{c}\text { Aplicación de las temáticas en actividades } \\
\text { laborales }\end{array}$} & Secado & $66,8^{a}(8,9)$ \\
\hline & Preservado & $60,8^{\mathrm{a}}(7,0)$ \\
\hline & Norma de calidad & $45,4^{b}(9,1)$ \\
\hline \multirow{3}{*}{$\begin{array}{l}\text { Interés de adquisición del conocimiento para } \\
\text { futuros proyectos }\end{array}$} & Secado & $93,7^{\mathrm{a}}(9,7)$ \\
\hline & Preservado & $90,9^{\mathrm{a}}(5,7)$ \\
\hline & Norma de calidad & $90,0^{\mathrm{a}}(10,1)$ \\
\hline \multirow{3}{*}{ Consideración del grado de dificultad del tema } & Secado & $42,6^{a}(7,6)$ \\
\hline & Preservado & $33,3^{a}(4,0)$ \\
\hline & Norma de calidad & $60,8^{a}(11,5)$ \\
\hline
\end{tabular}

Nota: valores entre paréntesis representa la desviación estándar; letras diferentes indican diferencias significativas con un $\alpha=0,05$

Análisis de adquisición de conocimientos y efectos de aprendizaje teórico y práctico

La evaluación de la curva del conocimiento adquirido cuantifico (CAC) (figura 1), mostró una misma tendencia en las tres temáticas, que consistió en un aumento del CAC a la finalización de cada curso, seguido por un decrecimiento posterior a 30 semanas; el aumento del CAC varió del 30 al $52 \%$, siendo la temática de la norma de calidad la que mostró mayor conocimiento adquirido (del 51,3\%), seguida por preservado (con 33,5\%) y en menor cantidad la de secado (con 20,4\%), posterior a 30 semanas se encontraron decrecimientos del 12 al $30 \%$ reiterando la 
tendencia que la norma de calidad presentó una pérdida del 34,6\% (una retención del 54,7\%), seguida por la el tema de preservado con un 23,3\% (retención del 62,9\%) y secado con apenas un $11 \%$ de decrecimiento (retención del 73,4\%).

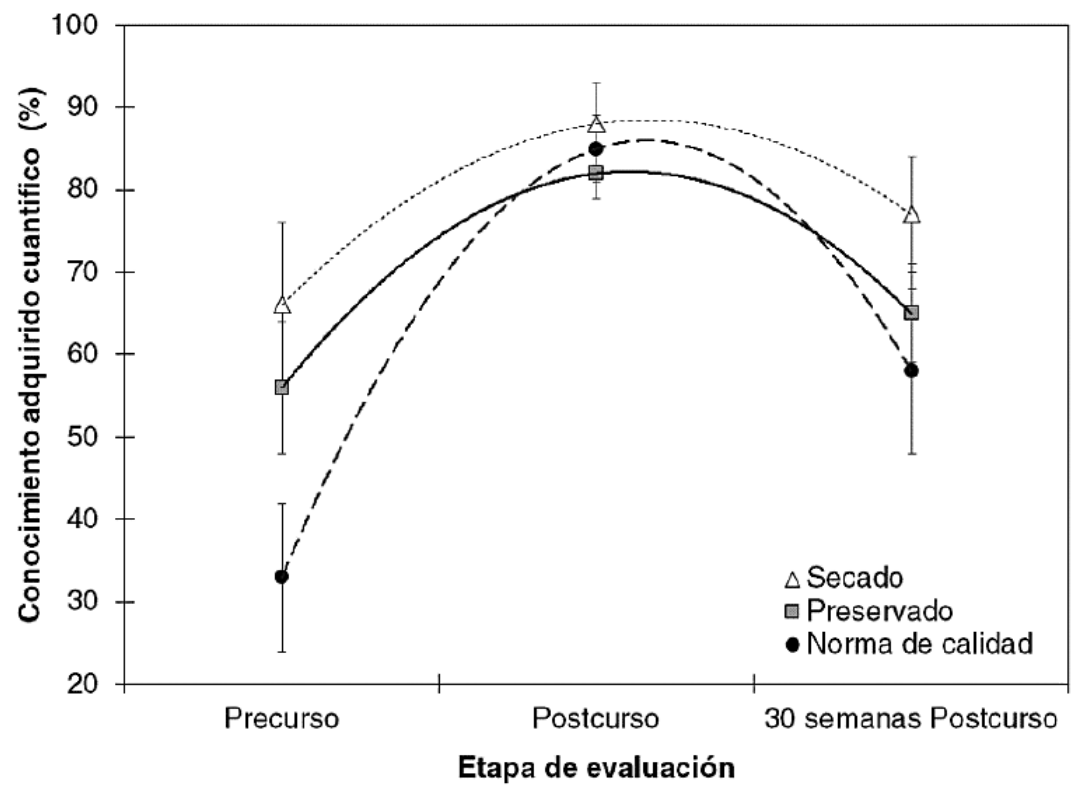

Figura 1. Curva de conocimiento adquirido cuantifico (CAC) en la etapa de Precurso, Postcurso y 30 semanas Postcurso con tres temas de aprendizaje forestal a cinco grupos de estudio.

Al analizar la composición del CAC a partir de los valores de aprendizaje cuantificado (VAC) teóricos y prácticos (cuadro 3), se encontró que la curva de temática de secado presentó el menor aumento en el conocimiento, los valores del VAC teórico y práctico no mostraron diferencias significativas entre la fase post curso y 30 semanas post curso, lo que significa que el proceso de evaluación incidió en una buena asimilación del conocimiento. Respecto al conocimiento en la norma de calidad el VAC teórico y práctico muestran diferencias significativas del conocimiento adquirido, la impartición del curso aumentó el conocimiento; sin embargo, posteriormente se dio la pérdida de retención, el valor a 30 semanas post curso no llegó a ser tan bajo como el pre curso (inferiores al 46\%) siendo eficiente de manera parcial el sistema empleado, mostrando que si se mantuvo la permanencia del conocimiento en los usuarios. El VAC teórico del tema de preservado muestra como el sistema de aprendizaje utilizado incrementó significativamente el conocimiento adquirido después de impartido el curso, y este presentó una disminución significativa posterior a las 30 semanas post curso; sin embargo, la retención del conocimiento en comparación con la valoración pre curso fue beneficiosa y significativa, caso contrario los valores portados para el VAC práctico de preservado, en el cual la pérdida de conocimiento a las 30 semanas adquiere valores similares al conocimiento poseído previo del cuso, siendo el sistema utilizado ineficiente.

Análisis perceptual del método de aprendizaje implementado

La percepción del método significativo-observacional empleado vario significativamente en las tres temáticas (figura 2), la temática de secado obtuvo la mejor percepción $\left(r^{2}\right.$ de 0,82), al considerar que se impartió con una utilidad y simplicidad elevada, siendo altamente funcional, lo cual incide con los resultados de la figura 1 y cuadro 2, en los cuales se muestra como la 
retención del conocimiento fue mayor; en cambio con la temática de preservado se mantuvo la percepción que el sistema presentó una utilidad elevada pero con una tendencia mayor a ser considerado como complicado, esta percepción no fue homogénea y por ende el coeficiente de determinación fue menor que el obtenido en secado $\left(r^{2}\right.$ de 0,61$)$. Por otra parte, en la temática de la norma de calidad no se observa una tendencia perceptual clara, al tener una respuesta heterogénea, incidiendo en un coeficiente de determinación muy bajo ( $r^{2}$ de 0,43 ), aunque la tendencia muestra ser de utilidad elevada y simple, una cantidad significativa de usuarios opinaron lo contrario, lo que no permitió observar una tendencia clara.

Cuadro 3. Valores de aprendizaje cuantificado (VAC) teórico y práctico obtenido en las tres fases de evaluación con tres temáticas industriales forestales.

\begin{tabular}{|c|c|c|c|}
\hline \multirow{2}{*}{ Temática } & Fase de Evaluación & \multicolumn{2}{|c|}{ VAC $(\%)$} \\
\cline { 2 - 4 } & Precurso & Teórico & Práctico \\
\hline \multirow{3}{*}{ Secado } & Postcurso & $55,5^{\mathrm{a}}(6,5)$ & $78,5^{\mathrm{a}}(7,0)$ \\
\cline { 2 - 4 } & $35,1^{\mathrm{b}}(4,0)$ & $92,2^{\mathrm{b}}(8,8)$ \\
\cline { 2 - 4 } & Po semanas Postcurso & $70,7^{\mathrm{b}}(6,9)$ & $84,4^{\mathrm{b}}(6,7)$ \\
\hline \multirow{3}{*}{ Preservado } & Precurso & $38,3^{\mathrm{a}}(6,0)$ & $74,2^{\mathrm{a}}(5,7)$ \\
& Postcurso & $70,0^{\mathrm{b}}(8,6)$ & $93,3^{\mathrm{b}}(6,7)$ \\
\hline \multirow{3}{*}{ Norma de } & Pemanas Postcurso & $65,5^{\mathrm{c}}(8,8)$ & $75,0^{\mathrm{a}}(4,3)$ \\
\cline { 2 - 4 } & Precurso & $22,9^{\mathrm{a}}(6,5)$ & $45,3^{\mathrm{a}}(4,6)$ \\
\cline { 2 - 4 } & Postcurso & $75,7^{\mathrm{b}}(7,7)$ & $90,2^{\mathrm{b}}(6,7)$ \\
\cline { 2 - 4 } & 30 semanas Postcurso & $51^{\mathrm{c}}(9,0)$ & $62,0^{\mathrm{c}}(8,0)$ \\
\hline
\end{tabular}

Nota: valores entre paréntesis representa la desviación estándar; letras diferentes indican diferencias significativas con un $\alpha=0,05$

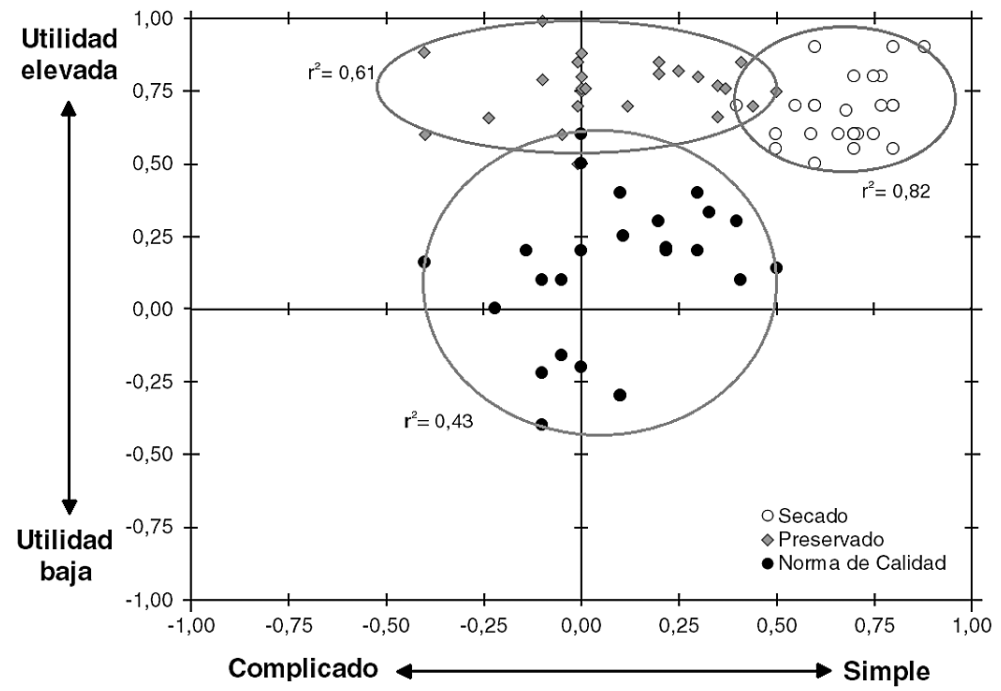

Figura 2. Agrupaciones perceptuales obtenidas en la valoración de tres temas de aprendizaje forestal a cinco grupos de estudio. 
Al descomponer esta percepción en la sección teórica y práctica del curso (figura 3) se destaca una mejor precepción grupal en la sección práctica (figura 3b) en comparación a la teórica que presentó valores en los ejes negativos. Al analizar la temática de secado en ambas secciones del sistema de aprendizaje empleado, presenta percepciones superiores a 0,20, considerada como muy útil y simple; para el tema de preservado se mantiene para ambas secciones la percepción de utilidad elevada; sin embargo, la sección teórica mostró una tendencia a ser complicada, lo cual indica que el sistema requiere ajustes en la parte teórica, al no ser tan aceptada como la sección práctica. Finalmente, con la temática de la norma de calidad la alta heterogeneidad de los datos no permite visualizar una tendencia clara, si bien la sección práctica tiende a ser mejor calificada que la teórica, no es significativa $\left(r^{2}\right.$ de 0,39$)$, lo que implica que el sistema utilizado no se adecua completamente a este tipo de temática, requiriendo ajustes significativos en la sección práctica y en menor medida en la teórica.
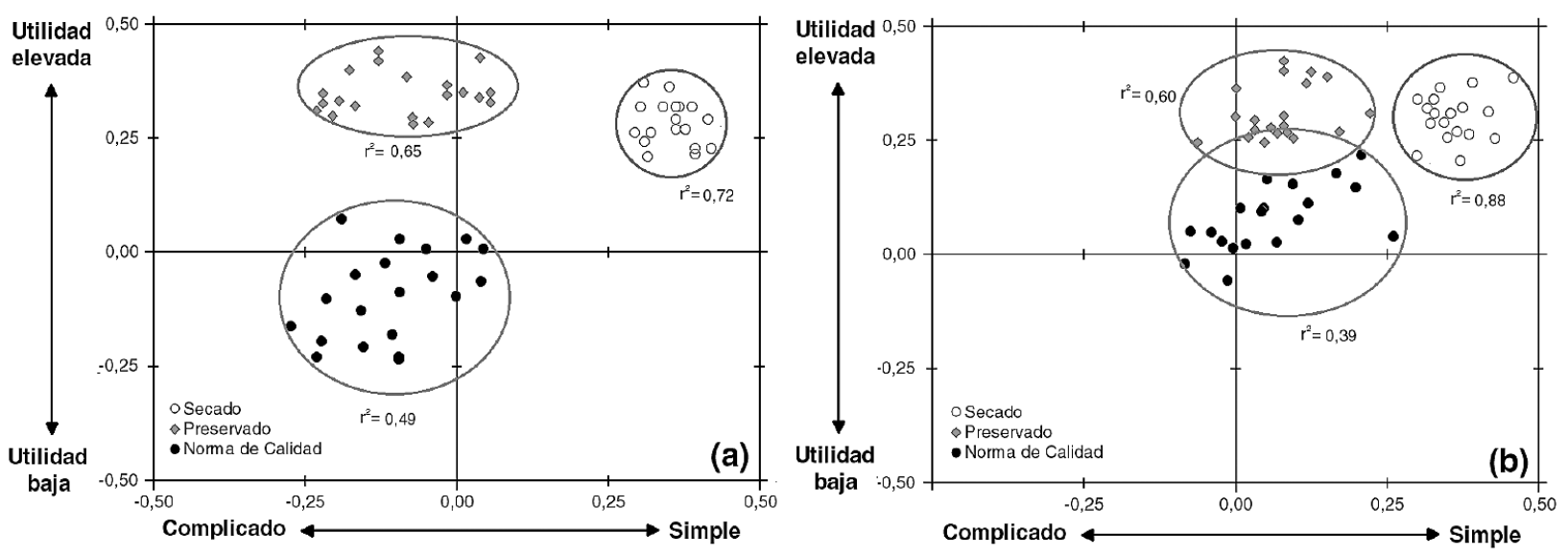

Figura 3. Agrupaciones perceptuales obtenidas en la valoración teórica (a) y práctica (b) de tres temas de aprendizaje forestal a cinco grupos de estudio.

Respecto a la temática de la norma de calidad, la cual presentó la menor retención, evidenciando la necesidad de mejorar o replantear el sistema de aprendizaje evaluado, [11], [13], [15] destaca que pérdidas de retención superiores al $20 \%$ en menos de 6 meses demuestra que el sistema de aprendizaje utilizado es complicado o no genera la atracción o desarrollo suficiente para que el usuario pueda tener mayor capacidad de interpretación.

El segundo argumento muestra como la variabilidad de adquisición del conocimiento está influenciado por su aplicabilidad en aspectos laborales, [14] enfatizan que un elemento fundamental del sistema de aprendizaje significativo-observacional, es que el usuario pueda aplicar o reintegrar a sus actividades diarias la mayoría de los conocimientos adquiridos, esto debido a que el procesamiento de reintegración de información es la constante reiteración de un concepto o elemento que facilita la capacidad de retención de la información, por esta razón en varias temáticas el aspecto práctico (cuadro 3) presentó mejores retenciones en comparación con la parte teórica, al implementar los usuarios en sus actividades laborales los temas de secado y preservado, les resulta más fácil asimilar y reintegrar mejor la información práctica que los conceptos teóricos que son menos utilizados, [1], [4] mencionan que en este tipo de actividades en las cuales el elemento práctico tiene más reintegración que el elemento teórico, se debe promover en el usuario un análisis crítico de la actividad en la cual de forma consiente reincorpore el concepto teórico y balancee la retención de información. 
El tercer factor mencionado por [11], [13], [15] es la relación funcionalidad-tiempo, este concepto se basa en la capacidad de desarrollar el conocimiento adquirido en el tiempo [2], conforme el tema sea funcional y no solo permita al usuario implementarlo sino incentivarlo a aumentar su conocimiento, con el tiempo el manejo de la temática será mejor y presentará mayor fluidez neuronal en el momento de aplicar el conocimiento. La poca relación de funcionalidad-tiempo genera que el conocimiento se pierda en el tiempo y su potencialidad de uso disminuya, [1]-[3] destacan en este aspecto que temas complicados o de percepción compleja (cuadro 1) deben ser explotados en funcionalidad para mantenerse en el tiempo, de lo contrario la retención será mínima, razón por la cual el tema de la norma de calidad muestra menores valores de aprendizaje, menor retención y mayor pérdida de retención y expectativa de aprendizaje, el sistema empleado en conjunto a su poca implementación laboral generan funcionalidad baja en el tiempo provocando no ser recordado de forma adecuada por los usuarios, caso contrario mostraron los temas de secado y preservado de la madera, los cuales por su impacto y uso constante en sus actividades laborales tienden a ser funcionales en el tiempo.

\section{Puntos a considerar en el proceso de aprendizaje en temáticas forestales}

Es importante destacar el principio de variabilidad educativa de temas paralelos, que presentan [4], [5] que consiste en temas con funcionalidad similar, pero con conceptos distintos, en los cuales se puede usar un sistema de aprendizaje análogo, pero con modificaciones en los puntos de mayor desgaste de aprendizaje (reconsiderados como conceptos o actividades complicadas); en el caso de este proyecto, los temas de secado, preservado y norma de calidad de la madera se presintieron eran temas similares, por lo cual el sistema de aprendizaje implementado fue funcional entre sí; sin embargo, la percepción por parte de los usuarios no fue conforme, el tema secado y preservado son paralelos por su común aplicabilidad en las actividades labores, en cambio la norma de calidad no presenta el mismo patrón (figura 3) ya que presentó mayor complejidad y una utilidad menor, [4] mencionan como en estos casos se deben hacer modificaciones y analizar la carga de conceptos teóricos y prácticos, analizar las actividades prácticas desarrolladas y el manejo conceptual implementado, ya que temas con percepción variable no garantizan la utilización de los conceptos adquiridos a largo plazo y muestran pérdidas de retención superiores al 70 \%.

Todo lo anterior sugiere que en temas forestales es necesario hacer un análisis y replanteamiento de las técnicas empleadas, ya que existe una alta posibilidad de que su efecto sea mínimo o nulo, como destaca[9], indicando que los temas forestales deben ser impartidos con claridad, al desearse que su impacto sea elevado en el tiempo por tratarse de temas en recursos naturales y sostenibilidad ambiental, [4] menciona la necesidad de mejorar aspectos como, el reforzamiento extensionista, el manejo de conceptos claros, el buen balance de conceptos teóricos y prácticos, evaluaciones subjetivas que permitan definir las fortalezas y debilidades del proceso empleado a partir del cual se pueda mejorar el sistema empleado y finalmente el proceso de la constante actualización y reforzamiento de conceptos forestales adquiridos. Esto con el fin de mejorar la capacidad y retención del conocimiento de los usuarios meta en el tiempo.

\section{Conclusiones}

La aplicación del sistema de aprendizaje significativo-observacional en temáticas forestales mostró respuestas distintas, con el tema secado se presentó una mayor retención (superior al $70 \%$ ) y desarrollo de aprendizaje, lo cual incidió que el sistema implementado se interpretara como muy útil y simple; caso similar con la temática de preservado que mostró un aprendizaje con una retención superior al 62,9 \% y una percepción final del tema de moderadamente simple 
pero muy útil, estos resultados se debieron al conocimiento previos de los usuarios, en conjunto con la posibilidad de aplicación de conceptos en actividades diarias que incidieron en que estos conceptos sean funcionales en el tiempo.

Contrario a lo mencionado, la temática de norma de calidad presentó menor funcionalidad con el sistema de aprendizaje utilizado, que provocó menor retención del conocimiento (56 \%) y una percepción variable del curso, debido a que su conocimiento previo fue bajo, al no aplicarse en sus labores diarias y su funcionalidad no fue la óptima, por cual es necesario hacer revaloraciones o cambios en el sistema empleado para aumentar la retención y funcionalidad del tema.

Se recomienda para estudios futuros realizar valoraciones post curso un año después del proceso de enseñanza, con la finalidad de conocer la retención a largo plazo del conocimiento brindado. Asimismo, es importante cuantificar el impacto de implementar los cambios propuestos en este estudio sobre la metodología de enseñanza de la temática abordada con el fin de mejorar las técnicas de aprendizaje.

\section{Agradecimientos}

Se agradece a la Vicerrectoría de Investigación y Extensión (VIE) del Instituto Tecnológico de Costa Rica por el apoyo para el desarrollo de esta investigación.

\section{Referencias}

[1] C. Barrantes and J. Yagüe, "Adults' Education and Agricultural Innovation: A Social Learning Approach," Procedia - Social and Behavioral Sciences, vol. 191, pp. 163-168, 2015.

[2] A. McElwain, V. Finnegan, A. Whittaker, J. Kerpelman, F. Adler-Baeder, and A. Duke, "Evaluation and lessons learned from an undergraduate service learning course providing youth-focused relationship education," Evaluation and Program Planning, vol. 58, pp. 116-124, 2016.

[3] Y. Kashiwaba, K. Kozawa, and H.-H. Uchida, Evaluation of learning environment for ESD (Education for Sustainable Development) using activity index of salivary amylase. 2012, pp. 1087-1090.

[4] S. Miller, R. Taylor-Piliae, and K. Insel, "The association of physical activity, cognitive processes and automobile driving ability in older adults: A review of the literature," Geriatric Nursing, vol. 37, no. 4, pp. 313-320, 2016.

[5] N. Berasategi, I. Alonso, and G. Roman, "Service-learning and Higher Education: Evaluating Students Learning Process form their Own Percpective," Procedia - Social and Behavioral Sciences, vol. 228, pp. 424-429, 2016.

[6] M. Zhan, X. Xiang, and W. Elliott, "Education loans and wealth building among young adults," Children and Youth Services Review, vol. 66, pp. 67-75, 2016.

[7] M. Hei, J.-W. Strijbos, E. Sjoer, and W. Admiraal, "Thematic review of approaches to design group learning activities in higher education: The development of a comprehensive framework," Educational Research Review, vol. 18, pp. 33-45, 2016.

[8] B. Németh, "Research and Development of Adult Education through Higher Education Institutions: A Challenge and Perspective for Better Adult Learning and Education," Procedia - Social and Behavioral Sciences, vol. 142, pp. 97-103, 2014.

[9] W. Silva, L. Silva, C. Silva, and P. Nascimento, "Optimization and simulation of drying processes using diffusion models: application to wood drying using forced air at low temperature," Wood Science and Technology, vol. 45, no. 4, pp. 787-800, 2011.

[10] F. Obounou-Akong, P. Gérardin, M.-F. Thévenon, and C. Gérardin-Charbonnier, "Hydrogel-based boron salt formulations for wood preservation," Wood Science and Technology, 2015.

[11] E. Aponte, "Towards a new evaluation culture in higher education," Prospects, vol. 28, no. 3, pp. 393-399, 1998.

[12] B. Wahlgren, K. Mariager-Anderson, and S. Sørensen, "Expanding the traditional role of the adult education teacher - The development of relational competences and actions," Teaching and Teacher Education, vol. 60, pp. 303-311, 2016. 
[13] A. Prisặcariu, "Approaches of Quality Assurance Models on Adult Education Provisions," Procedia - Social and Behavioral Sciences, vol. 142, pp. 133-139, 2014.

[14] S. Hubackova and I. Semradova, "Research Study on Motivation in Adult Education," Procedia - Social and Behavioral Sciences, vol. 159, pp. 396-400, 2015.

[15] R. Schendel and T. McCowan, "Expanding higher education systems in low- and middle-income countries: the challenges of equity and quality," Higher Education, vol. 72, no. 4, pp. 407-411, 2016.

[16] S. Cakula, A. Jakobsone, and M. Florea, "Automated Learning Support System for Adult Education Institutions and Enterprises," Procedia Computer Science, vol. 77, pp. 191-198, 2015.

[17] A. McElwain, V. Finnegan, A. Whittaker, J. Kerpelman, F. Adler-Baeder, and A. Duke. "Evaluation and lessons learned from an undergraduate service learning course providing youth-focused relationship education, " Evaluation and Program Planning, vol. 58, pp.116-124, 2016.

[18] N. Berasategi, N. Alonso, and G. Roman. Service-learning and Higher Education: Evaluating Students Learning Process form their Own Percpective. Procedia - Social and Behavioral Sciences, vol. 228, pp. 424-429, 2016.

[19] S. Miller, R. Taylor-Piliae, and K. Insel, K. "The association of physical activity, cognitive processes and automobile driving ability in older adults: A review of the literature". Geriatric Nursing, vol. 37, núm. 4, pp. 2016.

[20] Y. Kashiwaba, K. Kozawa, and H. Uchida. "Evaluation of learning environment for ESD (Education for Sustainable Development) using activity index of salivary amylase," Higher Education, vol. 5, pp. 1087-1090, 2012.

[21] FAO, "Propuesta de Educación Forestal," Septiembre 2016. (En línea). Disponible en: http://www.fao.org/forestry/material-educativo/es/

[22] INTE 06-07-02-2014. Uso general de la madera. Primera Edición. INTE CTN 06. San José, Costa Rica. 37 p. 2014

[23] INTE 06-07-01-2011. Madera estructural -Clasificación en grados estructurales para la madera aserrada mediante una evaluación visual. Primera Edición. INTE CTN 06. San José, Costa Rica. 37 p. 2011

[24] R. Moya, F. Muñoz, J. Salas, A. Berrocal, L. Leandro, E. Esquivel. "Tecnología de madera de plantaciones forestales: Fichas Técnicas”. Revista Forestal Mesoamericana Kurú, vol. 7, num.18-19, p.:207. 2010. 